${ }^{1}$ Facultad de Ciencias de la Actividad Física y del Deporte, Universidad de Playa Ancha, Valparaíso, Chile.

${ }^{2}$ Facultad de Ciencias del Deporte, Departamento Educación Física y Deportiva Universidad de Granada, España.

${ }^{3}$ Grupo de Investigación CTS-642 IDAFISAD. Facultad Ciencias del Deporte, Universidad de Granada, España.

${ }^{4}$ Facultad de Educación, Universidad Católica de la Santísima Concepción, Chile. aprofesor de Educación Física, MSc. Actividad Física y Salud.

Becario de doctorado de la Facultad Ciencias del Deporte, Universidad de Granada, España.

bProfesor de Educación Física, Doctor en Educación Física por la Universidad de Granada.

cProfesor de Educación Física, Doctor en Educación Física por la Universidad de Málaga.

Recibido el 24 de marzo de 2014, aceptado el 6 de mayo de 2015 .

Correspondencia a: Hernaldo Carrasco Beltrán hernaldo.carrasco@upla.cl

\section{Efecto de los juegos reducidos en la composición corporal y la condición física aeróbica en un grupo de adolescentes escolares}

\author{
HERNALDO CARRASCO BELTRÁN ${ }^{1,3, a}$, RAFAEL E. REIGAL GARRIDO ${ }^{2,3, c,}$, \\ DAVID ULLOA DÍAZZ,a, IGNACIO JESÚS CHIROSA RÍOS ${ }^{2,3, \mathrm{~b}}$, \\ LUIS JAVIER CHIROSA RÍOS $2,3, \mathrm{~b}$

\section{Effects of small-sided exercises on body composition and maximal oxygen uptake in adolescents}

Background: The regular practice of physical activity contributes to weight control and improves maximal oxygen uptake $\left(\mathrm{VO}_{2 \max }\right)$, socialization and quality of life. Aim: To determine the effect of moderate-to-vigorous physical activity on body composition and $\mathrm{VO}_{2 \max }$ in a group of overweight school-age males. Material and Methods: We studied fifty-five overweight males aged $15.6 \pm 0.7$ years. Participants were divided in two groups (experimental and control groups). The intervention in the experimental group was the practice of small-sided games during 60 minutes, two days per week and during 11 weeks. At baseline and the end of the intervention, body composition was measured using bioimpedance and $V O_{2 \max }$ was calculated using the Course Navette test. Results: A 7\% reduction in the percentage of body fat and a $9 \%$ increase in $V_{2 \max }$ were observed in the experimental group $(p<0.01)$. No changes were recorded in the control group. Conclusions: Eleven weeks of small-sided exercises performed twice per week increased $\mathrm{VO}_{2 \max }$ and reduced fat mass in overweight adolescents.

(Rev Med Chile 2015; 143: 744-750)

Key words: Adolescents; Body Composition; Exercise; Motor Activity.

\section{L} os niveles de actividad física (AF) han disminuido en niños y adolescentes en la última década $^{1,2}$. Este fenómeno ha generado una gran preocupación, dado que bajos niveles de $\mathrm{AF}$ son un factor de riesgo de enfermedades cardiovasculares y metabólicas ${ }^{3,4,5}$. En Chile, la prevalencia de adolescentes obesos aumentó en los últimos 20 años ${ }^{6}$ específicamente, en la región de Valparaíso, los estudiantes de educación media presentan $26 \%$ de sobrepeso y $13,4 \%$ de obesidad en damas y $21,6 \%$ de sobrepeso y $16 \%$ de obesidad en varones. El 90\% de ellos presenta un bajo nivel en condición física ${ }^{7}$ a pesar de haber implementado una serie de programas de intervención, que no han tenido los resultados esperados.

La relación inversa entre la AF y porcentaje de masa grasa corporal (\%MG) ha sido suficientemente abordada en adolescentes ${ }^{2,13,14}$. De igual manera, los jóvenes que presentan altos niveles de AF tienen mayor consumo de oxígeno que la población sedentaria y un menor riesgo de padecer enfermedades no transmisibles ${ }^{1}$. La evidencia científica ha establecido que la AF moderada-alta intensidad se relaciona a un perfil lipídico-metabólico más saludable y a un menor riesgo de padecer enfermedades cardiovasculares ${ }^{8}$, mejores 
percepciones de salud, autoconcepto y calidad de vida $^{9,10,11}$ y a un funcionamiento cognitivo más eficaz ${ }^{12}$.

En un estudio, realizado por Weintraub y cols ${ }^{17}$ en niños, se observó que un programa deportivo basado en el fútbol durante 6 meses fue eficaz para el control del peso. Asimismo, Calcaterra $\mathrm{y}$ cols ${ }^{18}$ efectuaron un estudio con un grupo de adolescentes obesos, en el que observaron que la intervención a través de varios deportes colectivos durante 12 semanas fue adecuada para mejorar la composición corporal y los niveles de consumo de oxígeno.

En este sentido, en los deportes colectivos se emplean juegos de versión reducida, conocidos como los juegos reducidos (JR). En ellos se alteran elementos del juego real, como las dimensiones del terreno, el número de jugadores, las reglas o el tiempo disponible, aunque manteniendo la lógica interna del juego ${ }^{19-21}$. Diversas investigaciones han demostrados que los JR son un tipo de entrenamiento tan efectivo como el interválico ${ }^{22-24}$. Sin embargo, tiene la ventaja que permite aportar una mayor especificidad ${ }^{25} \mathrm{y}$ una optimización del tiempo de entrenamiento ${ }^{26}$. Además, incrementa el número de intervenciones y decisiones a tomar ${ }^{19,27}$.

En función de la intensidad y tipo de ejercicio, los JR, específicamente el 3 vs.3, alcanzan intensidades cercanas a $80 \%$ y $90 \%$ de la frecuencia cardiaca máxima $(\mathrm{FCmax})^{28}$. En este sentido, existe poca evidencia que muestre la respuesta del \%MG y consumo de oxígeno máximo $\left(\mathrm{VO}_{2 \max }\right)$ utilizando los JR en adolescentes no deportistas. La mayoría de las investigaciones ha incluido principalmente deportistas (amateur o elite), donde las adaptaciones y respuestas fisiológicas son mayores respecto a sujetos no deportistas ${ }^{29}$.

A la luz de los antecedentes descritos, y como estrategia para la promoción de la salud en edad escolar, el objetivo de esta investigación fue determinar el efecto de la AF de moderada a alta sobre las variables de composición corporal (peso, índice de masa corporal y \%MG) y $\mathrm{VO}_{2 \max }$ en un grupo de adolescentes escolares con sobrepeso.

\section{Material y Método}

\section{Diseño}

Se ha empleado un diseño cuasi experimental con grupo control no aleatorizado, con medidas de pre y post test. Los sujetos fueron asignados en dos grupos (grupo experimental [GE] y grupo control [GC]). El grupo experimental ejecutó 11 semanas de JR y el grupo control no tuvo intervención y se le recomendó que mantuviese sus hábitos de vida.

\section{Sujetos}

Un total de cincuenta y cinco $(n=55)$ adolescentes hombres (rango mínimo y máximo de edad), con sobrepeso ( +1 a $+1,9$ DS) según las tablas OMS, $2007^{30}$ participaron en la investigación. Ellos accedieron voluntariamente a una invitación abierta comunicada a través de reuniones y afiches. A todos los sujetos se les envió una carta a los padres, apoderados o tutores del centro escolar, los que dieron su consentimiento informado por escrito para participar del estudio.

Los sujetos eran alumnos de $1^{\circ}$ y $2^{\circ}$ medio del liceo mixto María Luisa Bombal de la ciudad de Valparaíso, Chile. No practicaban deporte a nivel competitivo, no presentaban enfermedad crónica conocida ni estaban en seguimiento médico. El estudio fue desarrollado considerando la Declaración de Helsinki (2000) y fue aprobado por el comité de ética de la Universidad de Playa Ancha, Valparaíso-Chile.

\section{Procedimiento}

El período intervención de 11 semanas se llevó a cabo en el liceo mixto María Luisa Bombal de la ciudad de Valparaíso. Todas las sesiones de JR se realizaron dos veces a la semana, en horario extraescolar, que era entre las 18 y $20 \mathrm{~h}$. El grupo control no tuvo ningún tipo de intervención y el profesor utilizó la misma metodología de clase de educación física que utilizaba, de acuerdo al plan de estudio. La adherencia fue de $90 \%$ grupo experimental. Del grupo control, $95 \%$ concurrieron a las citaciones.

Antes y después de la intervención, a todos los sujetos se les estimó el $\mathrm{VO}_{2 \max }\left(\mathrm{ml} \cdot \mathrm{kg}^{-1} \cdot \mathrm{min}^{-1}\right)$ a través del test de Course Navette según lo propuesto por Léger y $\operatorname{cols}^{32}$, la frecuencia cardiaca máxima individual (lpm) con pulsómetros (Polar ${ }^{\circledR}$ modelo S610, Finlandia) y el \%MG con un bioimpedanciómetro (modelo OMRON HBF-306, Japón).

$$
\mathrm{VO}_{2 \max }
$$

Para determinar la capacidad aeróbica máxima se utilizó un test de de ida y vuelta de 20 metros o test Course Navette ${ }^{30}$. $\mathrm{El} \mathrm{VO}_{2 \max }\left(\mathrm{ml} \cdot \mathrm{kg}^{-1} \cdot \mathrm{min}^{-1}\right)$ se 
estableció según las ecuaciones propuestas por Léger $\mathrm{y} \mathrm{cols} \mathrm{s}^{31}$, para este grupo etáreo, donde: $\mathrm{VO}_{2 \max }=$ $(31,025+3,238 \mathrm{~V}-3,248 \mathrm{E}+0,1536 \mathrm{VE})$, siendo V la velocidad alcanzada en la última etapa completada y E la edad del participante. Se dio por terminada la prueba cuando los sujetos se retiraban de forma voluntaria o cuando no eran capaces de mantener el ritmo impuesto según la señal sonora. En relación con la potencia aeróbica, medida a través del test de Course Navette, se clasificó según los rangos sugeridos por el manual de Fitnessgram ${ }^{33}$. Los niveles aceptables para las edades comprendidas entre los 14 y 17 años son de 39,70-42,40 ml/ $/ \mathrm{kg} /$ min y $41,30-44,10 \mathrm{ml} / \mathrm{kg} / \mathrm{min}$, respectivamente, de potencia aeróbica para su edad.

\section{Frecuencia cardiaca máxima individual}

Para determinar la FCmax individual, se registró durante toda la prueba de $\mathrm{VO}_{2 \max }$, considerándose como FCmax individual en valor (lpm) alcanzado al final de la prueba. La intensidad del entrenamiento se estableció como un porcentaje de la FCmax individual de acuerdo a 4 zonas de intensidades (75\% de la FCmax, $75-84 \%$ de la FCmax, $85-89 \%$ de la FCmax y $90 \%$ de la FCmax).

Para establecer el \%MG, se utilizó un bioimpedanciómetro con 4 electrodos en el mango. Para el análisis de la composición corporal se tomaron como referencia los percentiles de estatura por edad y peso, así como el índice de masa corporal (IMC) por edad de la Organización Mundial de la Salud (OMS, 2007).

De forma previa al período de intervención, el grupo experimental realizó dos sesiones de familiarización con los JR y con dimensiones del terreno de juego. La sesión por día de JR basados en el fútbol tenía una duración total de $75 \mathrm{~min}$. El diseño de los JR correspondió con un 3 vs.3. El área total fue de $300 \mathrm{~m}^{2}(20 \times 15 \mathrm{~m})$ y por jugador de $50 \mathrm{~m}^{2}$. Se realizaron dos partes de 30 $\mathrm{min}$, teniendo un descanso entre partes de $5 \mathrm{~min}$. Todas las sesiones estuvieron precedidas por un calentamiento de 10 min basados en juegos de activación y movilidad articular.

\section{Análisis estadístico}

Todas las variables se expresaron como media y desviación típica. La normalidad de las variables fue analizada utilizando el test de Kolmogorov-Smirnov. Se empleó la técnica $t$-Student para evaluar las diferencias basales. El test de Levene fue realizado para analizar el supuesto de homogeneidad de varianzas entre grupos. Se realizó un análisis de la varianza (ANOVA) bifactorial para cada medida antropométrica y de condición física salvo para el índice de masa corporal (IMC), siendo un primer factor el grupo con dos niveles (control y experimental) y un segundo factor la variable pre-post con dos niveles (valores previos y tras la intervención). Se estudiaron los efectos principales y la interacción entre variables, utilizándose el estadístico de Bonferroni para evaluar la significación. Para analizar los valores del IMC, por la falta de distribución normal se aplicaron técnicas no paramétricas ( $U$ de Mann-Whitney y Wilcoxon). El nivel de significación estadística se estableció en $\mathrm{p}<0,05$. Todos los análisis se realizaron utilizando un paquete estadístico (SPSS v.20, Illinois, EEUU).

\section{Resultados}

Un total de cincuenta y cinco $(\mathrm{n}=55)$ adolescentes hombres, con sobrepeso, participaron en la investigación, con edades de 15,62 \pm 0,68 años, presentaron una altura de $166,96 \pm 6,81 \mathrm{~cm}$; correspondiente a un promedio ZT/E de $-0,54 \pm$ 0,83 ; un IMC de 22,96. Al momento del ingreso al programa, y realizar la medición con bioimpedanciómetro, el \%MG fue de 21,22 \pm 7,39\%; en el test de Course Navette, la FCmax fue de 200,56 $\pm 7,72$ ppm lo que corresponde a 38/55 sujetos al $90 \%$ de la FCmax y el $\mathrm{VO}_{2 \max }$ fue de 40,75 $\pm 6,08$ $\mathrm{ml} \cdot \mathrm{kg}^{-1} \cdot \mathrm{min}^{-1}$ lo que corresponde a $39 / 55$ sujetos en los niveles aceptables de potencia aeróbica para su edad. Además, en la Tabla 1 se presentan los datos correspondientes a cada grupo (GC y GE), no existiendo diferencias significativas entre las medidas basales presentadas $(\mathrm{p}>0,05)$.

En la Tabla 1 se muestran los análisis descriptivos basales de diversos parámetros antropométricos y de condición física para los grupos control y experimental. Tras el programa, el grupo control obtuvo un valor para IMC de 23,25; \%MG de $22,84 \pm 7,81 \%$ y $\mathrm{VO}_{2 \max }$ de 39,69 $\pm 5,57$. Asimismo, el grupo experimental obtuvo un valor de IMC de 22,63; \%MG de 19,58 \pm 6,68\% y $\mathrm{VO}_{2 \max }$ de 45,32 $\pm 7,62$. A estas variables se les realizó la prueba de homogeneidad de varianza (Levene), la cual indica que no existía para las medidas de IMC en los valores pre test $(9,94 ; \mathrm{p}<0,01)$ y post test $(10,12$; 
Tabla 1. Medias y desviaciones típicas basales de las variables analizadas

\begin{tabular}{|lcc|}
\hline & $\begin{array}{c}\text { Grupo } \\
\text { control } \\
\text { (GC) } \\
\mathbf{M} \pm \mathbf{D T}\end{array}$ & $\begin{array}{c}\text { Grupo } \\
\text { experimental } \\
\text { (GE) } \\
\mathbf{M} \pm \mathbf{D T}\end{array}$ \\
\hline Altura $(\mathrm{cm})$ & $166,33 \pm 5,97$ & $167,57 \pm 7,58$ \\
\hline Puntaje Z (T/E) & $-0,59 \pm 0,77$ & $-0,50 \pm 0,90$ \\
\hline Puntaje Z (IMC/E) & $0,72 \pm 1,22$ & $0,77 \pm 0,73$ \\
\hline IMC $\left(\mathrm{kg} / \mathrm{m}^{2}\right)$ & $23,16 \pm 4,29$ & $22,77 \pm 2,55$ \\
\hline Masa grasa $(\%)$ & $22,40 \pm 7,95$ & $20,09 \pm 6,76$ \\
\hline VO ${ }_{2 m a x}\left(\mathrm{ml}^{\prime} \mathrm{kg}^{-1} \cdot \mathrm{min}^{-1}\right)$ & $39,73 \pm 5,58$ & $41,74 \pm 6,48$ \\
\hline $\mathrm{FCmax}(\mathrm{ppm})$ & $198,52 \pm 7,69$ & $202,54 \pm 7,35$ \\
\hline
\end{tabular}

Puntaje Z (T/E)=Z-score (Talla/Edad); Puntaje Z (IM$\mathrm{C} / \mathrm{E})=\mathrm{Z}$-score (IMC/Edad); IMC= Índice de masa corporal; $\mathrm{VO}_{2 \text { máx }}=$ Consumo máximo de oxígeno; $\mathrm{FC}_{\text {máx }}=$ Frecuencia cardiaca máxima.

$\mathrm{p}<0,01)$. Se calculó el valor de la mediana para la variable IMC para el grupo control (pre test $=$ 22,37 ; post test $=22,39$ ) y experimental (pre test $=22,75$; post test $=22,46$ ). Asimismo, en ambos grupos los datos presentaron valores adecuados de asimetría (entre -1,08 y 0,60), curtosis (entre $-1,23$ y 1,57 ) y normalidad (Kolmogorov-Smirnov) de los datos (entre 0,22 y 0,96). Los análisis de la varianza indicaron resultados positivos del programa de JR, siendo significativo el efecto de interacción en las medidas \%MG $\left(\mathrm{F}_{[1,53]}=18,15\right.$; $\left.\mathrm{p}<0,001 ; \eta^{2}=0,26 ; 1-\beta=0,99\right)$ y $\mathrm{VO}_{2 \max }^{[1,53]}\left(\mathrm{F}_{[1,53]}=\right.$ 13,$\left.53 ; \mathrm{p}<0,001 ; \eta^{2}=0,20 ; 1-\beta=0,95\right)$.

Las comparaciones simples entre grupos (Figuras 1 y 2) mostraron que no existieron diferencias entre las medidas pretest (control vs experimental), aunque sí entre las postest en $\mathrm{VO}_{2 \max }(-5,63$; $\mathrm{p}<0,01)$. Las comparaciones intragrupos indicaron que en el grupo control existieron diferencias entre las medidas basales y post-intervención en $\%$ MG $(-0,44 ; p<0,01)$, así como también en el grupo experimental en las variables \%MG $(0,51$; $\mathrm{p}<0,01)$ y $_{2 \max }(-3,58 ; \mathrm{p}<0,01)$. Los resultados indicaron que el $\mathrm{VO}_{2 \max }$ mejoró en $9 \%$ y se redujo el \%MG en los adolescentes en $7 \%$.

Los análisis efectuados para el IMC, intragrupos (Wilcoxon) y entregrupos (U de Mann-Whitney), indicaron que existieron diferencias significativas entre las medidas basales y post-intervención en el grupo experimental $(-2,27 ; \mathrm{p}<0,05)$ (Figura 3 ).

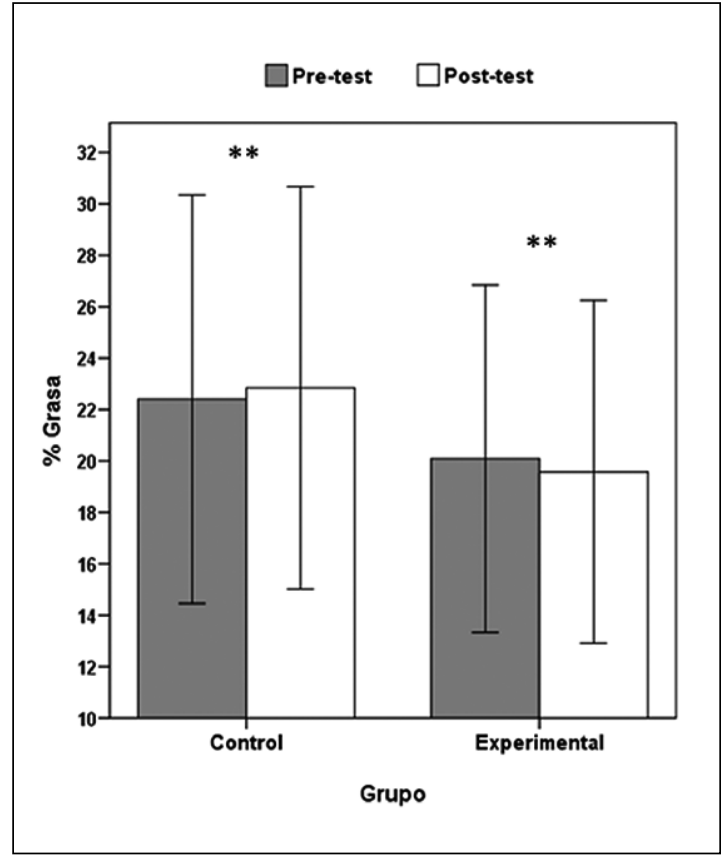

Figura 1. $\%$ Grasa $=\%$ masa grasa. ${ }^{*}$ Indica $p<0,01$.

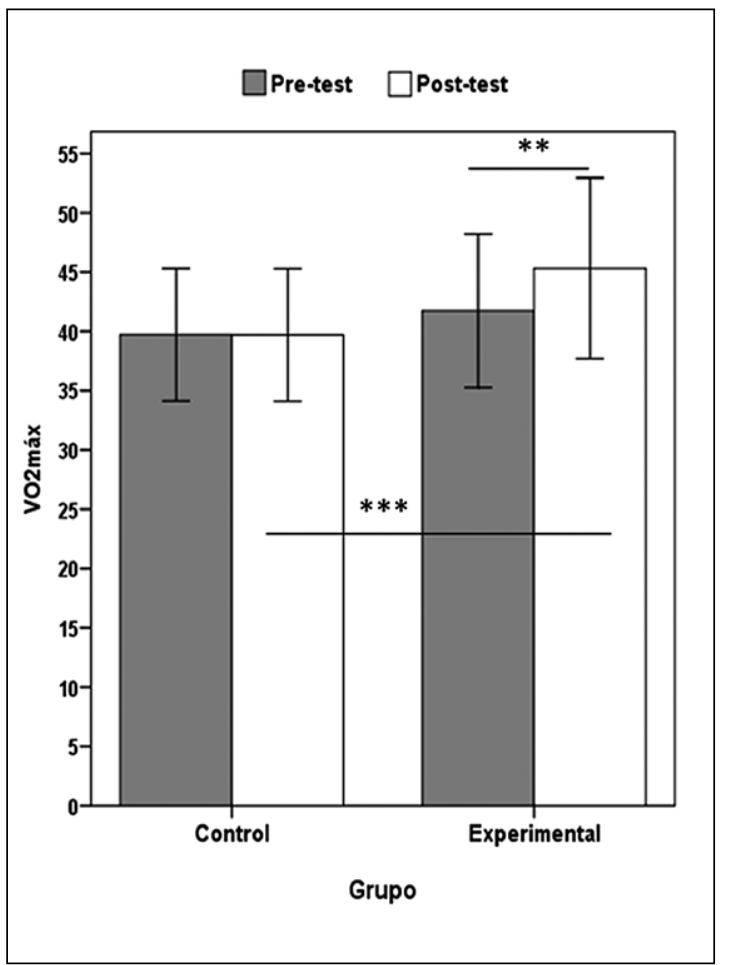

Figura 2. $\mathrm{VO}_{2 \max }=$ consumo máximo de oxígeno. ${ }^{* *}$ Indica $\mathrm{p}<0,01{ }^{* * *}$ Indica $\mathrm{p}<0,01$. 


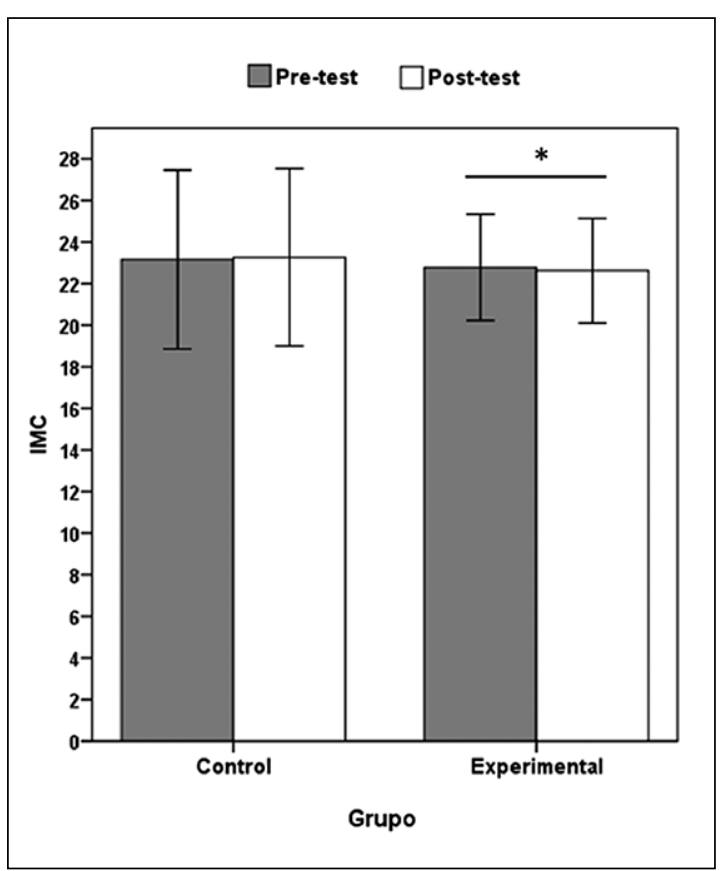

Figura 3. $I M C=$ índice de masa corporal. *Indica $p<0,05$.

\section{Discusión}

El objetivo principal de este estudio fue determinar el efecto de los JR sobre el \%MG y el $\mathrm{VO}_{2 \max }$ en adolescentes. Los principales hallazgos indicaron que tras 11 semanas de JR las variables relativas al \%MG y el $\mathrm{VO}_{2 \max }$ disminuyeron y aumentaron, respectivamente, en el grupo experimental a diferencia del grupo control.

Coincidiendo con los resultados obtenidos en nuestro estudio, Owen y cols ${ }^{34}$ señalaron que la prevalencia de actividad de baja intensidad provoca una mayor dependencia en el metabolismo aeróbico ${ }^{35,38}$, por otro lado, intercalando ejercicios de actividad de alta intensidades, como los presentados en los JR, se puede mejorar el $\mathrm{VO}_{2 \max }$ Conforme se describe en la literatura ${ }^{22}$ en referencia a este tipo de trabajo. Esto se alinea con los valores encontrados en este trabajo que están cercanos a los necesarios para mejorar el $\mathrm{VO}_{2 \max }^{23,36}$, por lo que los JR pueden ser una estrategia útil y efectiva para mejorar la potencia aeróbica máxima ${ }^{23,37}$.

La relación entre la obesidad y la actividad física ha sido un tema muy estudiado por el área de la salud cardiovascular ${ }^{1,2,37}$. Los resultados obtenidos en nuestro estudio muestran que en las variables de $\% \mathrm{MG}$ y el índice de masa corporal existieron cambios significativos entre las medidas pretest y postest en el grupo experimental. Desde el punto de vista de la salud, la relación entre el control del sobrepeso y el aumento de la condición física recientemente ha sido estudiado por London et $\mathrm{al}^{14}$, examinaron los efectos de los programas extraescolares basados en la AF en adolescentes, concluyendo que los adolescentes que participaban de estos programas reportaban mejor condición física y las tasas de obesidad eran más bajas. Estas conclusiones son similares a los del presente estudio, debido a que un programa de ejercicio físico de moderada a alta intensidad basada en los JR, tuvo resultados semejantes en las variables mencionadas anteriormente. Asimismo, los JR están asociados a ejercicios de alta intensidad ${ }^{39}$ y a un mayor gasto energético ${ }^{27,34}$. Se ha puesto de manifiesto que, durante los JR, la FCmax está mayor tiempo en las zonas de alta intensidad y se obtiene un mayor gasto energético ${ }^{27,34,39}$. Nuestros resultados muestran que, durante los JR (3 vs.3), se alcanzaron intensidades cercanas a $90 \%$ de la FCmax, coincidiendo con los resultados de Abrantes y cols ${ }^{28}$.

Los efectos de interacción en las medidas de

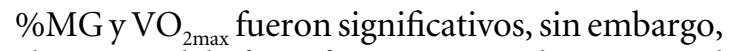
el tamaño del efecto fue mayor en el \%MG en el grupo experimental. En las comparaciones simples entre grupos (GC y GE) no existieron diferencias entre las medidas pretest en las variables estudiadas y únicamente en el $\mathrm{VO}_{2 \max }$ entre las medidas postest en el grupo experimental. Sin embargo, en las comparaciones intra grupos para la variable $\% \mathrm{MG}$ se encontraron diferencias significativas entre las medidas pretest y postest en el grupo experimental. Por ende, los JR parecen elevar los niveles de AF de moderada a alta intensidad contribuyendo al control del sobrepeso y al aumento de la condición física en adolescentes.

La escasa evidencia existente de la respuesta del $\% \mathrm{MG}$ y $\mathrm{VO}_{2 \max }$ utilizando los JR en adolescentes no deportistas ${ }^{29}$, nos ofreció grandes posibilidades de estimar los efectos sobre las variables estudiadas e implementar una intervención motivadora y efectiva para incrementar los niveles de $\mathrm{AF}^{2,40}$. La literatura señala que un mayor tiempo en las zonas de alta intensidad es consecuencia del número de participantes (3 vs. 3 o 4 vs.4).

Teniendo en cuenta las limitaciones impuestas por el tipo de metodología utilizada (por ejemplo cuasi-experimental) no podemos afirmar con 
exactitud que los cambios producidos son por el efecto de los JR, sin embargo, lo que puede estar claro, es la necesidad de generar un cambio de visión de las sesiones de Educación Física. Estas sesiones no deben quedarse en una mera descripción técnica de los diferentes deportes, sino ofrecer la posibilidad de realizar intervenciones en salud, incluyendo trabajos como los JR, en donde ha quedado patente, que producen un importante trabajo en la FC y por extensión, en el gasto cardiaco, $\% \mathrm{MG}$ y el $\mathrm{VO}_{2 \max }$.

Por lo tanto, sobre la base de los resultados podemos concluir que 11 semanas 2 veces a la semana de ejercicios basados en los JR pueden influenciar y modificar el nivel de AF en la vida diaria y nivel de salud. De esta forma, la estrecha relación entre la salud y la condición física queda plasmada en la mejora del $\mathrm{VO}_{2 \max }$ y la reducción en el \%MG en los adolescentes estudiados. Esta investigación se considera como una primera aproximación a la implementación de los JR en las clases de educación física, la mejora de la capacidad para realizar las tareas diarias con vigor y en la un búsqueda de un estado de completo bienestar (bio-psico-social), aspectos muy importantes en la salud de los niños. Desde este punto de vista, la eficacia de los JR (ejercicios de moderada a alta intensidad) se asocian a cambios significativos en los protectores de salud más relevantes (capacidad cardiovascular y control del sobrepeso).

Agradecimientos: A la Dirección General de Investigación, Universidad de Playa Ancha, Valparaíso. Chile.

\section{Referencias}

1. Ortega FB, Ruiz JR, Castillo MJ, Moreno LA, González-Gross M, Wärnberg J, et al. Bajo nivel de forma física en los adolescentes españoles. Importancia para la salud cardiovascular futura (Estudio AVENA). Revista Española de Cardiología 2005; 58 (8): 898-909.

2. Van der Horst K, Paw MJCA, Twisk JW, Van Mechelen W. A brief review on correlates of physical activity and sedentariness in youth. Medicine and Science in Sports and Exercise 2007; 39 (8): 1241.

3. Goñi E, Guillermo I. Actividad físico-deportiva, autoconcepto físico y satisfacción con la vida. European Journal of Education and Psychology 2010; 3 (2): 199-208.

4. Pontifex MB, Raine LB, Johnson CR, Chaddock L, Voss
MW, Cohen NJ, et al. Cardiorespiratory fitness and the flexible modulation of cognitive control in preadolescent children. Journal of Cognitive Neuroscience 2011; 23 (6): 1332-45.

5. Ekelund U, Jianan L, Lauren B, Dale W, Pippa G, Ashley $\mathrm{C}$, et al. Moderate to vigorous physical activity and sedentary time and cardiometabolic risk factors in children and adolescents. JAMA 2012; 307 (7): 704-12.

6. Reyes M, Díaz E, Lera L, Burrows R. Ingesta y metabolismo energético en una muestra de adolescentes chilenos con sobrepeso y obesidad. Rev Med Chile 2011; 139 (4): 425-31.

7. MINSAL-Chile. Situación Epidemiológica en Chile de las ENT y sus factores de riesgo. 2011. Disponible en http://seremi5.redsalud.gob.cl/wrdprss_minsal/wp content/uploads/2012/01/Epi-ENT.pdf [Consultado el 23 de marzo de 2014].

8. García-Artero E, Ortega FB, Ruiz JR, Mesa JL, Delgado M, González-Gross M, et al. El perfil lipídico-metabólico en los adolescentes está más influido por la condición física que por la actividad física (estudio AVENA). Revista Española de Cardiología 2007; 60 (6): 581-8.

9. Mota J, Santos RM, Silva P, Aires L, Martins C, Vale S. Associations between self-rated health with cardiorespiratory fitness and obesity status among adolescent girls. Journal of Physical Activity \& Health 2012; 9 (3): 378.

10. Padilla-Moledo C, Castro-Piñero J, Ortega FB, Mora J, Márquez S, Sjöström M, et al. Positive health, cardiorespiratory fitness and fatness in children and adolescents. The European Journal of Public Health 2012; 22 (1): 52-6.

11. Vélez A, Devon LG, Shawn MA. The impact of a 12week resistance training program on strength, body composition, and self-concept of Hispanic adolescents. The Journal of Strength \& Conditioning Research 2010; 24 (4): 1065-73.

12. Wu CT, Pontifex MB, Raine LB, Chaddock L, Voss MW, Kramer AF, et al. Aerobic fitness and response variability in preadolescent children performing a cognitive control task. Neuropsychology 2011; 25 (3): 333.

13. Fung C, Kuhle S, Lu C, Purcell M, Schwartz M, Storey $\mathrm{K}$, et al. From best practice to next practice: the effectiveness of school-based health promotion in improving healthy eating and physical activity and preventing childhood obesity. International Journal of Behavioral Nutrition and Physical Activity 2012; 9 (1): 27.

14. London RA, Oded G. Afterschool program participation, youth physical fitness, and overweight. American Journal of Preventive Medicine 2013; 44 (3): S200-7.

15. Taverno R, Dowda M, Beets MW, Pate RR. Physical Activity Behavior and Related Characteristics of Highly Active Eighth-Grade Girls. Journal of Adolescent Health 2013; 52 (6): 745-51. 
16. Berg K. Justifying physical education based on neuroscience evidence. Journal of Physical Education, Recreation \& Dance 2010; 81 (3): 24-46.

17. Weintraub DL, Tirumalai EC, Haydel KF, Fujimoto M, Fulton JE, Robinson TN. Team Sports for Overweight Children: The Stanford Sports to Prevent Obesity Randomized Trial (SPORT). Archives of Pediatrics \& Adolescent Medicine 2008; 162 (3): 232-7.

18. Calcaterra V, Larizza D, Codrons E, De Silvestri A, Brambilla P, Abela S, et al. Improved metabolic and cardiorespiratory fitness during a recreational training program in obese children. Journal of Pediatric Endocrinology and Metabolism 2013; 26 (3-4): 271-6.

19. Duarte R, Batalha N, Folgado H, Sampaio J. Effects of exercise duration and number of players in heart rate responses and technical skills during futsal small-sided games. Open Sports Sci J 2009; 2: 37-41.

20. Hill-Haas S, Coutts A, Dawson B, Rowsell G. Time-motion characteristics and physiological responses of small-sided games in elite youth players: the influence of player number and rule changes. The Journal of Strength \& Conditioning Research 2010; 24 (8): 2149-56.

21. Parlebas P. Léxico de praxiología motriz. España, Paidotribo. Ltda. 2001. p. 138-43.

22. Hill-Haas SV, Rowsell GJ, Dawson BT, Coutts AJ. Acute physiological responses and time-motion characteristics of two small-sided training regimes in youth soccer players. The Journal of Strength \& Conditioning Research 2009; 23 (1): 111-5.

23. Impellizzeri FM, Marcora SM, Castagna C, Reilly T, Sassi A, Iaia FM, et al. Physiological and performance effects of generic versus specific aerobic training in soccer players. International Journal of Sports Medicine 2006; 27 (6): 483-92.

24. Dellal A, Chamari K, Pintus A, Girard O, Cotte T, Keller D. Heart rate responses during small-sided games and short intermittent running training in elite soccer players: a comparative study. The Journal of Strength \& Conditioning Research 2008; 22 (5): 1449-57.

25. Reilly T, Morris T, Whyte G. The specificity of training prescription and physiological assessment: A review. Journal of Sports Sciences 2009; 27 (6): 575-89.

26. Little T, Alun GW. Suitability of soccer training drills for endurance training. The Journal of Strength \& Conditioning Research 2006; 20 (2): 316-9.

27. Casamichana D, Julen C, Alexandre D. Influence of different training regimes on physical and physiological demands during small-sided soccer games: continuous vs intermittent format. The Journal of Strength\& Conditioning Research 2013; 27 (3): 690-7.

28. Abrantes CI, Nunes MI, MaÇãs VM, Leite NM, Sampaio JE. Effects of the number of players and game type constraints on heart rate, rating of perceived exertion, and technical actions of small-sided soccer games. The Journal of Strength \& Conditioning Research 2012; 26 (4): 976-81.

29. Dellal A, Hill-Haas S, Lago-Penas C, Chamari K. Small-sided games in soccer: amateur vs professional players' physiological responses, physical, and technical activities. The Journal of Strength \& Conditioning Research 2011; 25 (9): 2371-81.

30. Léger LA, Mercier D, Gadoury C, Lambert J. The multistage 20 metre shuttle run test for aerobic fitness. Journal of Sports Sciences 1988; 6 (2): 93-101.

31. Bulletin of the World Health Organization 2007; 85: 660-7.

32. Léger LA, Lambert J, Goulet A, Rowan C, Dinelle Y. Capacité aérobie des Québécois de 6 á 17 ans -test navette de 20 métres avec paliers de 1 minute. Canadian Journal of Applied Sport Sciences 1984; 9 (2): 64-9.

33. The Cooper Institute for Aerobics Research. FITNESSGRAM test administration manual. Champaign, IL: Human Kinetics, 1999. Disponible en: http://www. cooperinstitute.org [Consultado el 11 de julio de 2014].

34. Owen AL, Wong DP, McKenna M, Dellal A. Heart rate responses and technical comparison between small-vs large-sided games in elite professional soccer. The Journal of Strength \& Conditioning Research 2011; 25 (8): 2104-10.

35. Reilly T, David B. The net physiological cost of dribbling a soccer ball. Research Quarterly for Exercise and Sport 1984; 55 (3): 267-71.

36. Helgerud J, Lars CE, Ulrik W, Jan H. Aerobic endurance training improves soccer performance. Medicine and Science in Sports and Exercise 2001; 33 (11): 1925-31.

37. Fanchini M, Azzalin A, Castagna C, Schena F, Mccall A, Impellizzeri FM. Effect of bout duration on exercise intensity and technical performance of small-sided games in soccer. The Journal of Strength \& Conditioning Research 2011; 25 (2): 453-8.

38. Álvarez C, Olivo J, Robinson O, Quintero J, Carrasco V, Ramírez R, et al. Respuesta hipotensiva de la presión sistólica y diastólica a una sesión de ejercicio aeróbico en niños, adolescentes y adultos. Rev Med Chile 2013; 141 (11): 1363-70.

39. Rampinini E, Impellizzeri FM, Castagna C, Abt G, Chamari K, Sassi A, et al. Factors influencing physiological responses to small-sided soccer games. Journal of Sports Sciences 2007; (25): 659-66.

40. Cradock AL, Barrett JL, Carter J, McHugh A, Sproul J, Russo ET, et al. Impact of the Boston Active School Day Policy to Promote Physical Activity Among Children. American Journal of Health Promotion 2014; 28 (3): 54-64. 